\title{
Diagnostic accuracy of urinary survivin mRNA expression detected by RT-PCR compared with urine cytology in the detection of bladder cancer: A meta-analysis of diagnostic test accuracy in head-to-head studies
}

\author{
LIANG FU ${ }^{1}$, JIWANG ZHANG ${ }^{1}$, LING LI $^{1}$, YUXING YANG ${ }^{2}$ and YONGQIANG YUAN ${ }^{1}$ \\ ${ }^{1}$ Clinical Laboratory, Yongchuan Hospital of Chongqing Medical University, Chongqing 402160; \\ ${ }^{2}$ Department of Pathology, People's Hospital of Xinjiang Uygur Autonomous Region, \\ Urumchi, Xinjiang 830001, P.R. China
}

Received May 29, 2019; Accepted November 1, 2019

DOI: $10.3892 / \mathrm{ol} .2019 .11227$

\begin{abstract}
Survivin is a promising marker for the diagnosis of bladder cancer. The accuracy and clinical value of urinary survivin mRNA expression were compared with urine cytology, which is the standard diagnostic method for bladder cancer. Scientific databases, including PubMed, Web of Science, Cochrane Library and China National Knowledge Infrastructure, were searched in order to find studies that examined urinary survivin mRNA expression and urine cytology in the diagnosis of bladder cancer. Quality assessment was performed using the Quality Assessment of Diagnostic Accuracy Studies 2 tool in Revman 5.3 and data analysis was conducted using Stata/MP. The $\mathrm{I}^{2}$ statistic was used to evaluate heterogeneity and Deeks' funnel plot was generated to assess the possibility of publication bias. A total of 15 studies that evaluated a total of 1,624 patients were included in the present meta-analysis. The pooled sensitivity and specificity values for the detection of urinary survivin mRNA expression in the diagnosis of bladder cancer were 0.86 [95\% confidence interval (CI), 0.81-0.90] and 0.95 (95\% CI, 0.93-0.96), respectively. Regarding urine cytology, the pooled sensitivity and specificity values were 0.42 (95\% CI, 0.36-0.48) and 1.00 (95\% CI, 0.98-1.00), respectively. Furthermore, the differences in pooled sensitivity were statistically significant in the diagnosis of grade 1 and 2 bladder tumors. Summary receiver operating characteristic curve values for urinary survivin mRNA expression and urine cytology were 0.95 (95\% CI, 0.93-0.97) and 0.86 (95\% CI, 0.83-0.89), respectively. Urinary survivin mRNA expression was also more
\end{abstract}

Correspondence to: Mr. Yongqiang Yuan, Clinical Laboratory, Yongchuan Hospital of Chongqing Medical University, 439 Xuanhua Road, Chongqing 402160, P.R. China

E-mail: s2yyyq@163.com

Key words: bladder cancer, survivin mRNA, urine cytology, diagnosis, meta-analysis accurate compared with other diagnostic indicators, including positive likelihood ratios, negative likelihood ratios, diagnostic odds ratios and Youden's index. Compared with traditional urine cytology, urinary survivin mRNA detection using reverse transcription-PCR was identified to be more effective in the diagnosis of early bladder cancer.

\section{Introduction}

According to the Global Cancer Statistics 2018, bladder cancer is the tenth most common form of cancer and the ninth leading cause of cancer-associated mortality worldwide (1). At initial diagnosis, $\sim 70 \%$ of patients with bladder cancer exhibit non-muscle invasive bladder cancer; however, over a 5-year period the risk of recurrence has been identified to vary between 30 and $80 \%$, and $\sim 15 \%$ of cases of bladder cancer progress to muscle invasion (2). Therefore, early diagnosis and long-term surveillance of patients with bladder cancer are crucial. The current diagnostic and postoperative surveillance strategies are based on the combination of cystoscopy and voided urine cytology (3). Although these two methods are regarded as the gold standard for the diagnosis of bladder cancer, they have certain disadvantages. Cystoscopy, which is an invasive procedure, results in high costs for the healthcare system and the discomfort of patients (4). Additionally, the symptoms and their duration after cystoscopy induced pain during urination (50\%), increased urinary frequency (37\%), visible hematuria (19\%) and infection $(3 \%)(5,6)$. Furthermore, this procedure has been demonstrated to exhibit low sensitivity in the detection of carcinoma in situ, and tumors may be missed due to its operator-dependent effectiveness (7). Overall sensitivity of urine cytology has been identified to range between 28 and $100 \%$, with a median of $44 \%$; however, this procedure exhibits high sensitivity for detecting high-grade tumors and low sensitivity for low-grade tumors (range, 4-31\%) (8). In addition, negative cytology does not exclude the presence of a tumor as cytological results are patient-dependent and may be hindered by several factors, including low cellular yield, urinary tract infections and stones (9). 
Great efforts have been made to develop new tests with high diagnostic efficiency and reproducibility and low cost for the non-invasive detection of bladder cancer. To date, numerous potential urinary biomarkers have been suggested and the use of molecular biomarkers for bladder cancer surveillance has demonstrated potential clinical applicability $(4,10)$. Examples of these biomarkers include nuclear matrix protein 22 , survivin, matrix metallopeptidase 9 , bladder tumor antigen, cytokeratin, urinary bladder carcinoma antigen and Cyfra 21-1 (11).

Survivin is the smallest inhibitor of apoptosis protein with a single amino-terminal BIR domain and carboxy-terminal Coiled Coil domain (12). Survivin is a critical regulator of mitosis, and an inhibitor of apoptosis, which promotes the proliferation of tumor cells, induces angiogenesis and thus increases the invasion capacity of tumors (13). The expression of survivin is undetectable in terminally differentiated and mature tissues but is highly expressed in common types of human cancer, including lung, colon, pancreas, prostate and breast (14) cancer. Therefore, it is considered to be a new tumor marker, which may be useful in the diagnosis of human cancer (12). Swana et al (15) reported that survivin was expressed in $78 \%$ of patients with bladder cancer, as detected by immunohistochemistry (IHC), but was absent in normal bladder urothelium. Smith et al (16) detected the expression of survivin protein and mRNA in urine samples from patients with bladder cancer by Bio-Dot immunoassay and reverse transcription-PCR (RT-PCR), respectively, in 2001. In the following years, certain studies assessed the detection of survivin protein in urine samples using IHC, ELISA or Bio-Dot immunoassay as a means of diagnosing bladder cancer. The detection of urinary survivin expression has been identified by Bio-Dot immunoassay to be an accurate diagnostic method for bladder cancer that retains its efficiency regardless of tumor stage and grade (17). In addition to the survivin protein, the survivin gene has gradually gained interest as a marker for the diagnosis and treatment of bladder cancer. An increasing number of studies have examined the expression of survivin mRNA in urine by RT-PCR for the diagnosis of bladder cancer. A meta-analysis by Liang et al (18) concluded that both survivin protein and mRNA may be used as biomarkers for bladder cancer detection, and survivin RNA exhibited higher accuracy compared with survivin protein. In addition, numerous studies have demonstrated the various accuracy of RT-PCR detection of urinary survivin mRNA expression in the diagnosis of bladder cancer. Weikert et al (19) reported a sensitivity of $68.6 \%$ and a specificity of $100 \%$ was identified in 53 patients with bladder cancer. Pu et al (20) reported a sensitivity of $90.4 \%$ and a specificity of $96.6 \%$ for the diagnosis of bladder cancer. Eissa et al (21) reported a sensitivity of $76.1 \%$ and a specificity of $95.0 \%$ in 86 patients.

The aim of the present meta-analysis was to review and summarize the results of previous experimental studies confirming the potential diagnostic value of urinary survivin mRNA as a marker for bladder cancer, and to compare this test by RT-PCR with traditional cytology. In addition, the present study aimed to assess the quality of published studies.

\section{Materials and methods}

Search strategy. The present meta-analysis was performed according to the Preferred Reporting Items for Systematic Reviews and Meta-Analyses guidelines (22). Scientific databases, including PubMed, Web of Science, Cochrane Library and China National Knowledge Infrastructure (CNKI), were comprehensively searched for publications between January 2001 and January 2019 to identify studies on the use of urinary survivin mRNA expression and urine cytology in the diagnosis of bladder cancer. The published literature search was conducted in English and restricted to original research studies. Published studies in the CNKI database were searched using Chinese-language characters, since this database contains research papers published in Chinese. The following terms, which are Medical Subject Headings key words, were searched in the text, title or abstract of relevant studies: 'Bladder cancer' or 'carcinoma of bladder' or 'urothelial carcinoma of the urinary tract' and 'survivin'. Similar publications identified in the reference lists of the retrieved studies were also obtained.

Selection criteria. The retrieved studies were independently reviewed by two reviewers, who agreed on which studies were eligible for the present meta-analysis; discrepancies were discussed and resolved by consensus. The following inclusion criteria were applied to the published studies retrieved by the database search: i) Studies published in English or Chinese; ii) studies that included a $2 \times 2$ contingency table; iii) urinary survivin mRNA expression detected by RT-PCR; iv) urine cytology as a comparison test and v) cystoscopy and/or histopathology used as the gold standard. Furthermore, the following exclusion criteria were applied: i) Case reports, case series and review studies; ii) animal or cell experiments; iii) use of immunohistochemical staining or western blot detection of survivin; iv) patients with urinary tract tumors other than bladder cancer or v) incomplete clinical data.

Data extraction and quality assessment. The following primary outcome data were extracted from the studies included in the meta-analysis: i) True positives (TP); ii) false positives (FP); iii) false negatives (FN); iv) true negatives (TN); and v) the total number of patients enrolled in each study. Additional data included: i) The name of the first author; ii) publication year; iii) country; iv) study design and v) gold standard. The Quality Assessment of Diagnostic Accuracy Studies 2 (QUADAS-2) tool (23) was used to rate the quality of each of the included studies. This method consisted of four components, the selection of cases, trials to be assessed, gold standards and the flowcharts and progress of cases. Each of the assessments comprised seven items and the corresponding responses contained the terms 'yes', 'no' or 'uncertainty'. Positive answers ('yes') meant that the risk bias of a study was considered low, whereas negative ('no') and uncertain ('uncertainty') answers meant that the risk of bias was high.

Statistical analysis. Data analysis was performed using the midas (24-27) and metan [version 1.85; (28)] packages in Stata/MP (version 15.0; StataCorp, LLC). Revman 5.3 (version 5.3; The Nordic Cochrane Centre, The Cochrane Collaboration) was used for quality assessment. The sensitivity (Sen), specificity (Spe), positive likelihood ratios (PLRs), negative likelihood ratios (NLRs) and corresponding 95\% confidence intervals (CIs) were calculated using the TP, FP, FN and TN values, which were extracted from each 




Figure 1. Flow diagram of the literature search.

study prior to data pooling. The summary receiver operating characteristic curve (SROC) was constructed based on a bivariate regression approach and the pooled estimate for sensitivity and specificity was subsequently calculated. The diagnostic odds ratio (DOR) with 95\% CI and Youden's index $(\gamma=$ Sensitivity + Specificity-1) were also calculated. In addition, Fagan nomograms were generated to evaluate the clinical utility of the two diagnostic methods. Heterogeneity among the reports was assessed by the $\chi^{2}$ test (Cochran $Q$ test) and the $\mathrm{I}^{2}$ statistic. The DerSimonian Laird method for pooled analyses was used for $\mathrm{I}^{2}$ values $>50 \%$. Statistical heterogeneity was considered to be low when $\mathrm{I}^{2}$ was $25-49 \%$, moderate when $\mathrm{I}^{2}$ was $50-74 \%$ and high when $\mathrm{I}^{2}$ was $>75$ (29). In addition, Deeks' funnel plot asymmetry test based on parametric linear regression was used to identify the possibility of publication bias (25). As the primary aim of the present study was to assess the accuracy of urinary survivin mRNA expression in the early diagnosis of bladder cancer, Deeks' funnel plot was generated only for urinary survivin mRNA.

To compare urinary survivin mRNA expression with urine cytology, data of the pathological grade, stage and pooled sensitivity were collected. In addition, a paired $\chi^{2}$ test was performed for prime diagnostic indicators, sensitivity and specificity. A $\mathrm{Z}$ test was used to analyze the area under the curve (AUC) and Youden's index. $\mathrm{P}<0.05$ was considered to indicate a statistically significant difference.

\section{Results}

Search results and selected studies. The results of the current meta-analysis selection process are presented in Fig. 1. The initial search resulted in 959 studies, 490 of which were duplicates and were thus excluded. A total of two reviewers read the titles and abstracts of the remaining 469 studies; 428 irrelevant studies were removed by consensus. A total of 41 potentially eligible studies were selected; following reading the full text of each study and analyzing the results, 26 studies were excluded due to a lack of complete data or incomplete descriptions of the trials. A total of 15 eligible studies were included in the meta-analysis.

Included study characteristics. The main characteristics of the included studies are presented in Table I. The studies were from Germany (19), Kuwait (30), Portugal (31), Egypt $(21,32,33)$ and 
Table I. Characteristics of the studies included in the meta-analysis.

\begin{tabular}{|c|c|c|c|c|c|c|c|c|c|c|c|}
\hline \multirow[b]{2}{*}{ Author, year } & \multirow[b]{2}{*}{ Country } & \multirow[b]{2}{*}{ Gold standard } & \multicolumn{4}{|c|}{$\begin{array}{l}\text { Urinary survivin } \\
\text { mRNA expression }\end{array}$} & \multicolumn{4}{|c|}{ Urine cytology } & \multirow[b]{2}{*}{ (Refs.) } \\
\hline & & & $\mathrm{TP}$ & FP & FN & $\mathrm{TN}$ & TP & FP & FN & $\mathrm{TN}$ & \\
\hline Weikert et al, 2005 & Germany & Histopathology & 24 & 0 & 11 & 33 & 11 & 1 & 24 & 32 & (19) \\
\hline Pu et al, 2008 & China & Histopathology & 104 & 2 & 11 & 56 & 53 & 0 & 62 & 58 & (20) \\
\hline Pina-Cabral et al, 2007 & Portugal & Histopathology & 20 & 0 & 10 & 20 & 9 & 0 & 21 & 20 & (31) \\
\hline Al-Maghrebi et al, 2012 & Kuwait & Cystoscopy and histopathology & 70 & 1 & 10 & 24 & 32 & 1 & 48 & 24 & (30) \\
\hline Eissa et al, 2013 & Egypt & Cystoscopy and histopathology & 35 & 2 & 11 & 38 & 23 & 0 & 23 & 40 & (21) \\
\hline Eissa et al, 2010 & Egypt & Histopathology & 126 & 12 & 40 & 200 & 80 & 0 & 86 & 212 & (32) \\
\hline Eissa et al, 2010 & Egypt & Histopathology & 33 & 2 & 9 & 41 & 12 & 0 & 30 & 43 & (33) \\
\hline Jiang et al, 2006 & China & Histopathology & 32 & 2 & 3 & 48 & 18 & 0 & 17 & 50 & (34) \\
\hline Lin et al, 2007 & China & Histopathology & 45 & 1 & 3 & 29 & 14 & 0 & 34 & 30 & (35) \\
\hline Liu et al, 2009 & China & Histopathology & 53 & 4 & 7 & 16 & 15 & 0 & 45 & 20 & (36) \\
\hline $\mathrm{Pu}$ et al, 2008 & China & Histopathology & 60 & 1 & 4 & 19 & 26 & 0 & 38 & 20 & (37) \\
\hline Wan et al, 2008 & China & Cystoscopy and histopathology & 60 & 4 & 12 & 37 & 23 & 0 & 49 & 41 & (38) \\
\hline Wang et al, 2004 & China & Histopathology & 38 & 0 & 2 & 15 & 27 & 0 & 13 & 15 & (39) \\
\hline Wang et al, 2006 & China & Cystoscopy and histopathology & 47 & 1 & 1 & 15 & 28 & 0 & 20 & 16 & (40) \\
\hline Zhang et al, 2005 & China & Histopathology & 59 & 3 & 11 & 47 & 34 & 0 & 36 & 50 & (41) \\
\hline
\end{tabular}

TP, true positive; FP, false positive; FN, false negative; TN, true negative.

China (20,34-41), and eight of them were published in English. A total of 1,624 patients were included in the studies, 951 of which were diagnosed with bladder cancer. The remaining 673 control participants comprised healthy participants and patients with benign prostate hyperplasia, urinary tract infection, urethral stricture or urolithiasis and benign epithelial neoplasm of the bladder.

Quality assessment of the included studies. The quality assessment results of the included studies are presented in Fig. 2. Regarding patient selection, certain studies were identified to exhibit a high risk of bias since healthy patients were used as the control group, whereas others used non-malignant diseases of the urinary system as controls. The index test in the QUADAS-2 tool was associated with a high risk of bias, as study thresholds, reagents and procedure were often different. Regarding the reference standard, low risk of bias was identified. The flow and timing characteristic were associated with a low risk of bias.

Pooleddataforallincludedstudies. TableII presents thepairwise comparisons of prime diagnostic indicators between urinary survivin mRNA expression and urine cytology. The pooled Sen for urinary survivin mRNA expression and urine cytology were 0.86 (95\% CI, 0.81-0.90; Fig. 3) and 0.42 (95\% CI, 0.36-0.48; Fig. 4), respectively; the pooled Spe for urinary survivin mRNA expression and urine cytology were 0.95 (95\% CI, 0.93-0.96; Fig. 3) and 1.00 (95\% CI, 0.98-1.00; Fig. 4), respectively. The areas under the curve (AUC) for urinary survivin mRNA expression and urine cytology were 0.95 (95\% CI, 0.93-0.97; Fig. 5A) and 0.86 (95\% CI, 0.83-0.89; Fig. 5B). The summary estimates of DOR for urinary survivin mRNA expression



Figure 2. Assessment of the risk of bias of the included studies using the Quality Assessment of Diagnostic Accuracy Studies 2 tool. Presented is the proportion of studies with low (white), unclear (gray) and high (black) risk of bias. Each bar indicates the number and percentage of studies.

and urine cytology were 83.42 (95\% CI, 49.70-139.96) and 42.00 (95\% CI, 22.95-76.89), respectively; the pooled PLRs for urinary survivin mRNA expression and urine cytology were 16.4 (95\% CI, 11.6-23.2) and 178.5 (95\% CI, 25.1-1269.7) and the pooled NLRs were 0.14 (95\% CI, 0.10-0.20) and 0.59 (95\% CI, 0.53-0.65), respectively (Table II).

Fagan plot analysis was used to evaluate the differences in clinical utility between urinary survivin mRNA expression and urine cytology for the diagnosis of bladder cancer. Regarding the detection of urinary survivin mRNA expression, the probability of bladder cancer increased from 20 to $80 \%$ when the test was positive and decreased to $3 \%$ when the results were negative (Fig. 6A). For urine cytology, the probability of bladder cancer increased from 20 to $98 \%$ when the results were positive and decreased to $13 \%$ when the results were negative. The potential publication bias was evaluated by Deeks' funnel plot (Fig. 7). 
Table II. Comparisons of prime diagnostic indicators between the detection of urinary survivin mRNA expression and urine cytology.

\begin{tabular}{lclrr}
\hline Parameter & Urine survivin mRNA $(95 \% \mathrm{CI})$ & \multicolumn{2}{l}{ Urine cytology $(95 \% \mathrm{CI})$} & P-value \\
\hline Pooled Sen & $0.86(0.81-0.90)$ & 0.42 & $(0.36-0.48)$ & $<0.01$ \\
Grade & & & & $<0.01$ \\
G1 & $0.79(0.73-0.85)$ & 0.05 & $(0.01-0.12)$ & $<0.01$ \\
G2 & $0.84(0.79-0.88)$ & 0.35 & $(0.26-0.44)$ & $>0.05$ \\
G3 & $0.84(0.76-0.90)$ & $0.79(0.66-0.88)$ & $>0.05$ \\
Pooled Spe & $0.95(0.93-0.96)$ & $1.00(0.98-1.00)$ & $<0.05$ \\
$\gamma$ & 0.81 & 0.42 & - \\
PLR & $16.40(11.60-23.20)$ & $178.50(25.10-1269.70)$ & - \\
NLR & $0.14(0.10-0.20)$ & $0.59(0.53-0.65)$ & $>$ \\
DOR & $88.99(57.35-138.08)$ & $40.39(20.13-81.06)$ & $>0.05$ \\
SAUC & $0.95(0.93-0.97)$ & 0.86 & $(0.83-0.89)$ & \\
\hline
\end{tabular}

CI, confidence interval; PLR, positive likelihood ratio; NLR, negative likelihood ratio; $\gamma$, Youden's index; DOR, diagnostic odds ratio; Spe, specificity; Sen, sensitivity; -, not available; SAUC, summary area under the curve.

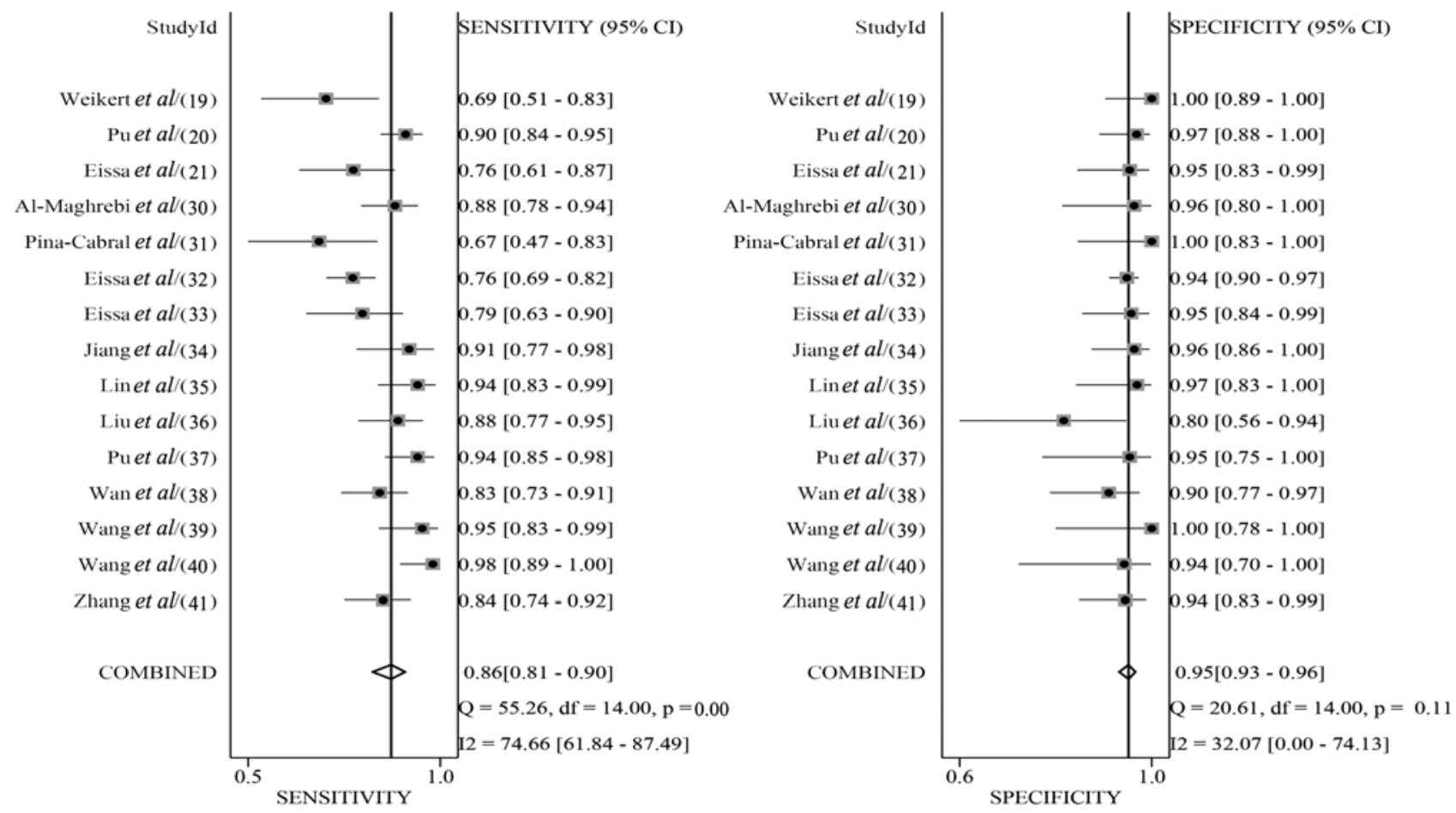

Urinary survivin mRNA

Figure 3. Forest plots demonstrating the sensitivity (left) and specificity (right) of urinary survivin mRNA expression. Squares indicate the estimates of sensitivity and specificity from each study; horizontal bars correspond to the $95 \%$ CIs; diamonds indicate the pooled sensitivity and specificity. CI, confidence interval; Q, Cochran $\chi^{2}$ test.

\section{Discussion}

Bladder cancer is a complex type of cancer with variable biological and clinical characteristics that has the tendency to recur and progress (42). Cystoscopy is 'gold standard' diagnostic procedure for bladder cancer, which can be invasive, expensive, and increase risk for urologic disease in patients $(43,44)$. Although urine cytology has high specificity (range, 85-100\%), it is associated with low overall sensitivity (range, 11-76\%) depending on tumor grade and is not suitable for preliminary screening in the diagnosis of bladder cancer (10). Survivin, which is a potential biomarker for urinary cancer, appears promising and worth validating in a prospective study with regards to its diagnostic and prognostic ability and clinical relevance $(4,10,45)$. Shariat et al $(17)$ have demonstrated that urinary survivin protein may be a predictor of high-grade 


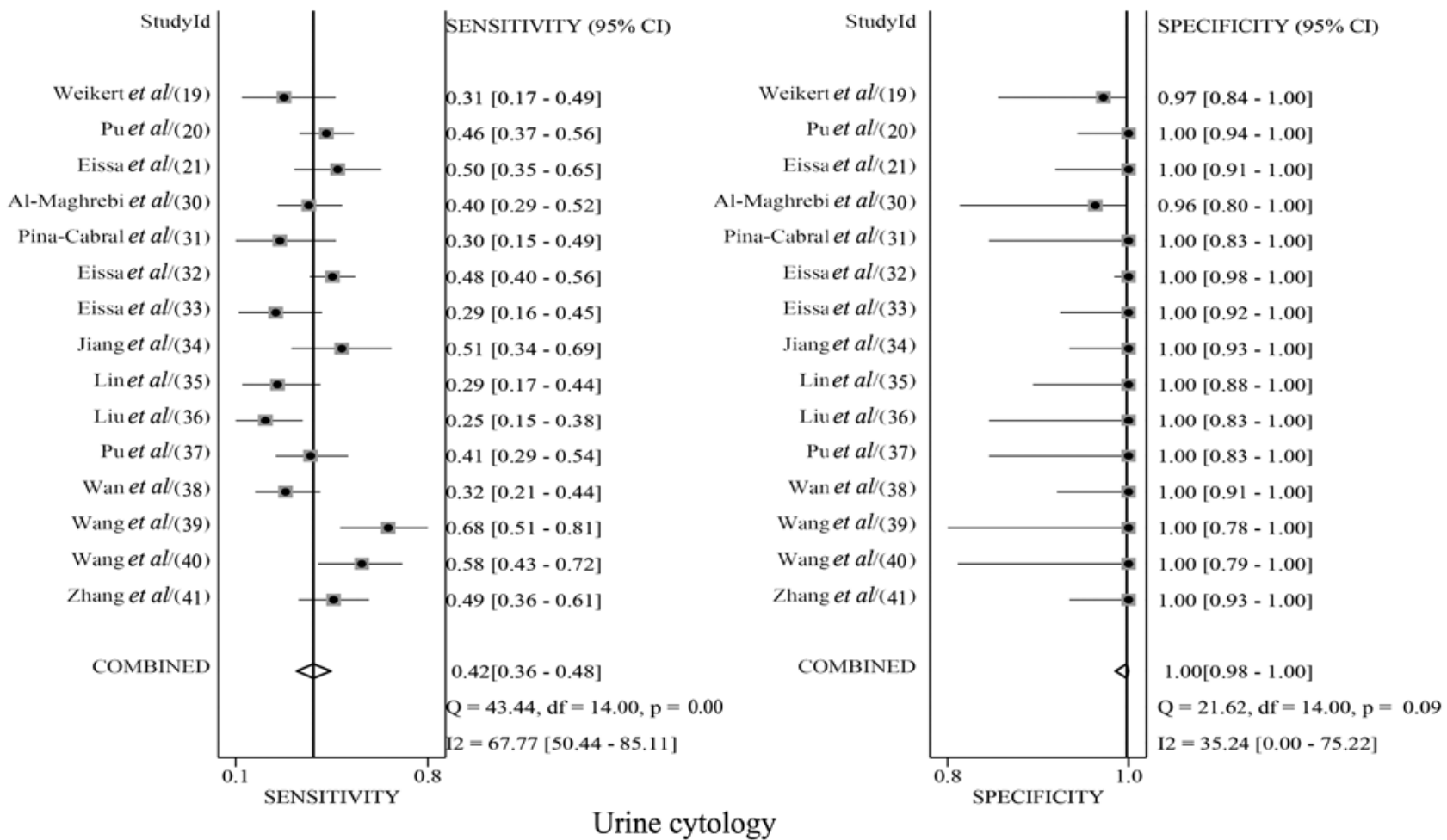

Figure 4. Forest plots demonstrating the sensitivity (left) and specificity (right) of urine cytology. Squares indicate the estimates of sensitivity and specificity from each study; horizontal bars correspond to the $95 \%$ CIs; diamonds indicate the pooled sensitivity and specificity. CI, confidence interval; Q, Cochran $\chi^{2}$ test.

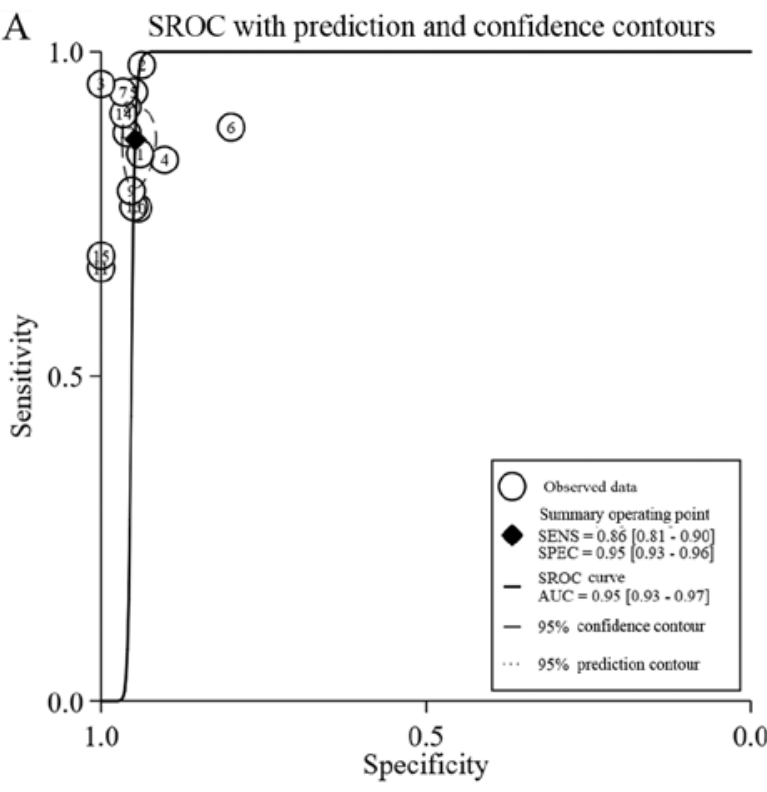

Urinary survivin mRNA

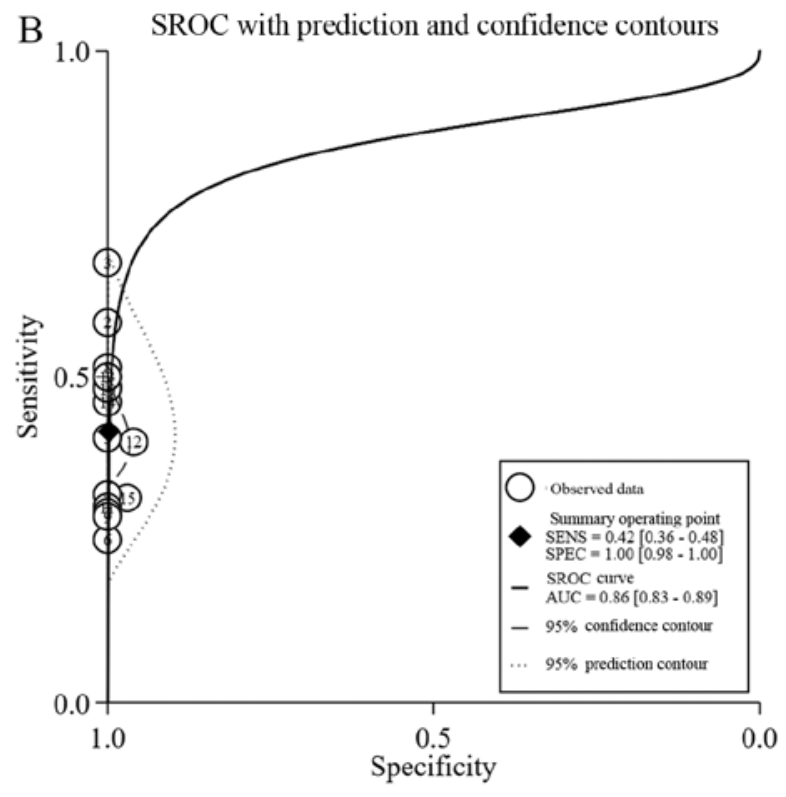

Urine cytology

Figure 5. SROC curves for (A) urinary survivin mRNA expression and (B) urine cytology. Circles represent each study. Demonstrated are SROC curves with confidence and prediction regions around the mean operating sensitivity and specificity point. SROC, summary receiver operating characteristic; SENS, sensitivity; SPEC, specificity; AUC, area under the curve.

bladder tumors and Weikert et al (19) have detected survivin mRNA expression in urine samples from $68.6 \%$ of patients with bladder cancer. Currently, there are several methods available to detect survivin expression, including IHC, ELISA and RT-PCR (46). Although IHC is regarded as a gold standard for the diagnosis of cancer, its use is restricted by the fact that tissue specimens are not easily obtained. Since survivin is a non-secreted short-lived protein, certain methods to detect its expression are based on the abundance of malignant cells in the urine sediment $(47,48)$. Although ELISA is considered a highly accurate method, it is limited to a certain extent due to being time-consuming and exhibiting poor uniformity (46). 




Urinary survivin mRNA

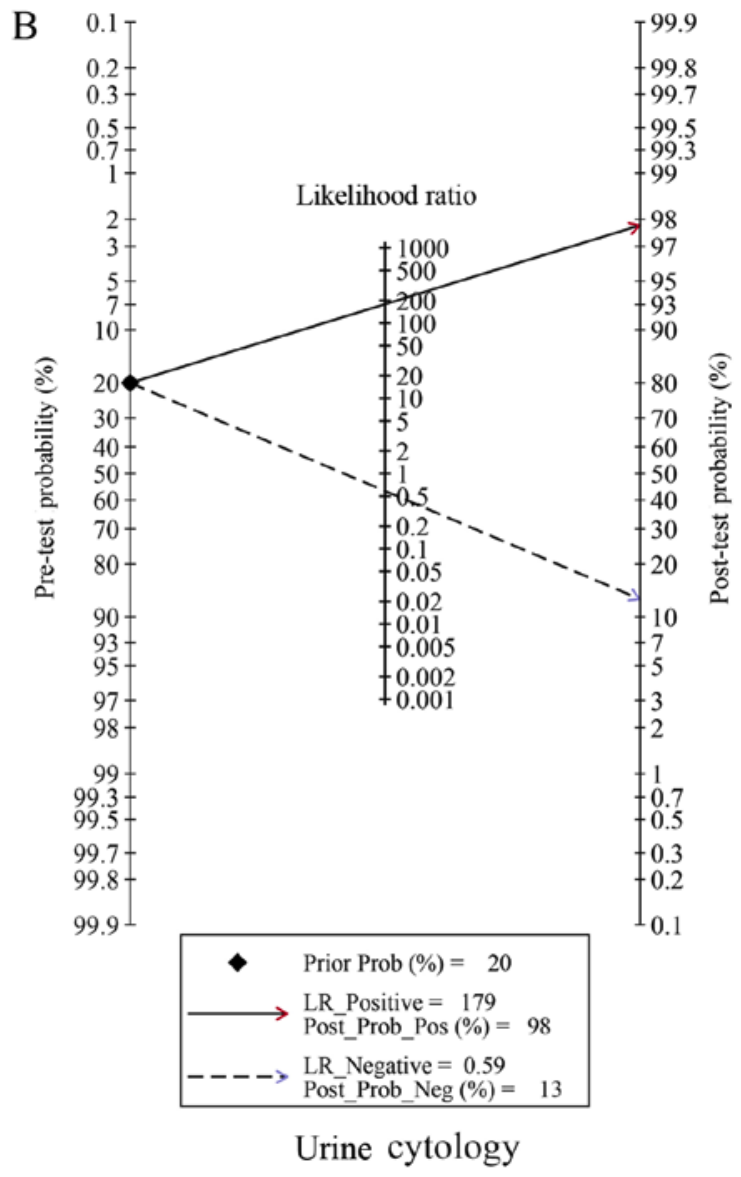

Figure 6. LR scattergrams for (A) urinary survivin mRNA expression and (B) urine cytology. A Fagan plot is included below each scattergram, demonstrating the relationship between the prior probability specified by the user (range, 0-1), the likelihood ratio (combination of sensitivity and specificity) and posterior test probability. LR, likelihood ratio.

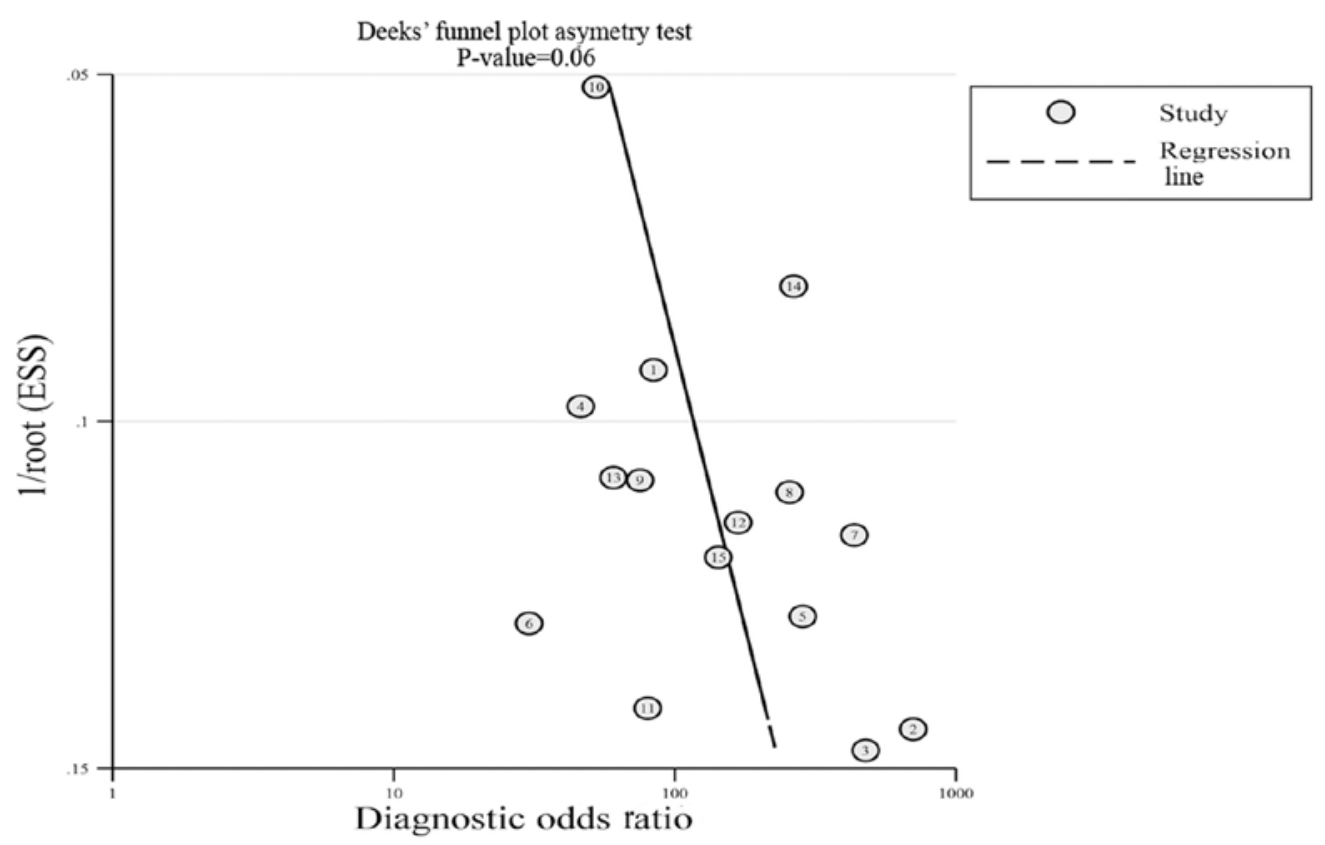

Figure 7. Deeks' funnel plot asymmetry test was performed to identify publication bias. The results indicated poor likelihood for publication bias in the present meta-analysis. Each solid circle represents a study in the meta-analysis. ESS, effective sample size.

In addition, protein-based assays are limited by insufficient antibody specificity and detection sensitivity lower compared with that of RT-PCR assays (49). RT-PCR exhibits very high sensitivity and is a well-established method able 
to exponentially amplify and quantify minuscule amounts of nucleic acids in malignant cells in the urinary sediment. Compared with RT-PCR, ELISA exhibits lower sensitivity (0.75; 95\% CI, 0.71-0.79) (18). The present study demonstrated that RT-PCR based assays may have high sensitivity, especially for the detection of the early stages of bladder cancer.

The aim of the present study was to perform a meta-analysis of published literature investigating urinary survivin mRNA expression detection by RT-PCR compared with urine cytology in the diagnosis of bladder cancer. The results demonstrated good diagnostic accuracy of urinary survivin mRNA expression and urine cytology for bladder cancer, but they showed their own different characteristics. The pooled sensitivity of urinary survivin mRNA expression was higher compared with that of urine cytology, and the difference was statistically significant in the diagnosis of grade 1 and 2 bladder tumors. These findings revealed that the expression of survivin may be associated with the degree of malignancy of bladder cancer, although its role in tumor metastasis and progression remains unclear. Regarding pooled specificity, urine cytology was higher compared with urinary survivin mRNA expression; as for SROC, urinary survivin mRNA expression was higher, but neither of these parameters were observed to exhibit statistically significant differences. Pooled PLRs $>10$ and pooled NLRs $<0.1$ were considered to provide convincing diagnostic evidence. PLRs $>5$ provide strong diagnostic evidence to rule in diagnoses; NLRs $<0.2$ provide strong diagnostic evidence to rule out diagnoses, in the majority of cases (50). In the present study, the NLR was 0.59 for urine cytology, which suggested that if the outcome for this method was negative, $59 \%$ of the patients may still have bladder cancer. However, the NLR for urinary survivin mRNA expression was 0.14 , indicating that $14 \%$ of patients may be misdiagnosed. Based on the assessed parameters from the retrieved studies, including Sen, Spe, AUC, PLR, NLR and DOR, the results of the present study demonstrated that survivin mRNA exhibited potential as a biomarker for the diagnosis of bladder cancer.

The present meta-analysis has several limitations. First, the results demonstrated that the heterogeneity between studies should not be ignored. Despite performing the subgroup and sensitivity analysis, heterogeneity was still detected. The DerSimonian Laird method was used for pooled analyses. The heterogeneity of the pooled studies was low to moderate in all analyses. This limitation is difficult to discuss since the individual studies in this meta-analysis contained different control groups. In previous studies, non-malignant diseases of the urinary system were assigned as the control groups $(37,38)$. In two of the studies, healthy participants were assigned to the control groups $(30,31)$. In other studies, healthy participants and non-malignant diseases of the urinary system were assigned as the control groups (19-21, 32-36, 39-41). Certain studies focused on diagnostics $(16,21,30,32,33)$, whereas others focused on the use of urinary mRNA expression as a tumor marker; however, there is not sufficient data to predict the recurrence of bladder cancer $(19,31)$. The pooled sensitivity and specificity for urinary survivin mRNA expression were 0.86 and 0.94 , respectively, indicating that the urinary survivin mRNA expression analysis via RT-PCR was accurate. Only studies written in English and Chinese were included; studies published in other languages were excluded, which may have led to further bias in this study. The experimental equipment and reagents of each laboratory and the threshold setting of urinary survivin mRNA expression may also vary.

Compared with traditional urine cytology, RT-PCR has the following advantages: i) Easy collection of urine samples; ii) high diagnostic accuracy; iii) low rate of missed diagnoses; and iv) simple procedure without discomfort for the patient. Furthermore, an important advantage of detecting urinary survivin mRNA expression using RT-PCR is its high sensitivity.

In summary, urinary survivin mRNA expression may serve as a screening marker for the diagnosis of bladder cancer. However, prior to its application in the clinical setting, a large prospective study is required in order to verify the accuracy of the RT-PCR assay and identify the optimal cut-off value clinical.

\section{Acknowledgements}

Not applicable.

\section{Funding}

The present study was funded by the Foundation of Yongchuan Hospital of Chongqing Medical University (grant nos. YJLCX201621 and 20180313).

\section{Availability of data and materials}

The datasets used and/or analyzed during the current study are available from the corresponding author on reasonable request.

\section{Authors' contributions}

JZ, YQY and LF designed the present study. YQY and LL analyzed the data and prepared the figures. LF and YXY performed the literature research and selected the relevant studies. LF and YQY wrote and revised the initial manuscript. All authors reviewed and approved the final version of the manuscript.

\section{Ethics approval and consent to participate}

Not applicable.

\section{Patient consent for publication}

Not applicable.

\section{Competing interests}

The authors declare that they have no competing interests.

\section{References}

1. Bray F, Ferlay J, Soerjomataram I, Siegel RL, Torre LA and Jemal A: Global cancer statistics 2018: GLOBOCAN estimates of incidence and mortality worldwide for 36 cancers in 185 countries. CA Cancer J Clin 68: 394-424, 2018.

2. Witjes JA and Hendricksen K: Intravesical pharmacotherapy for non-muscle-invasive bladder cancer: A critical analysis of currently available drugs, treatment schedules, and long-term results. Eur Urol 53: 45-52, 2008 
3. Babjuk M, Burger M, Zigeuner R, Shariat SF, van Rhijn BW, Compérat E, Sylvester RJ, Kaasinen E, Böhle A, Palou Redorta $\mathrm{J}$, et al: EAU guidelines on non-muscle-invasive urothelial carcinoma of the bladder: Update 2013. Eur Urol 64: 639-653, 2013

4. Kamat AM, Hegarty PK, Gee JR, Clark PE, Svatek RS, Hegarty N, Shariat SF, Xylinas E, Schmitz-Dräger BJ, Lotan Y, et al: ICUD-EAU International Consultation on bladder cancer 2012: Screening, diagnosis, and molecular markers. Eur Urol 63: 4-15, 2013.

5. Burke DM, Shackley DC and O'Reilly PH: The community-based morbidity of flexible cystoscopy. BJU Int 89: 347-349, 2002.

6. Biardeau X, Lam O, Ba V, Campeau L and Corcos J: Prospective evaluation of anxiety, pain, and embarrassment associated with cystoscopy and urodynamic testing in clinical practice. Can Urol Assoc J 11: 104-110, 2017.

7. Raitanen MP, Leppilahti M, Tuhkanen K, Forssel T, Nylund P and Tammela T; FinnBladder Group: Routine follow-up cystoscopy in detection of recurrence in patients being monitored for bladdercancer. Ann Chir Gynaecol 90: 261-265, 2001.

8. Lotan Y and Roehrborn CG: Sensitivity and specificity of commonly available bladder tumour markers versus cytology: Results of a comprehensive literature review and meta-analyses. Urology 61: 109-118, 2003.

9. Oeyen E, Hoekx L, De Wachter S, Baldewijns M, Ameye F and Mertens I: Bladder cancer diagnosis and follow-up: The current status and possible role of extracellular vesicles. Int J Mol Sci 20: pii: E821, 2019.

10. Lokeshwar VB, Habuchi T, Grossman HB, Murphy WM, Hautmann SH, Hemstreet GP III, Bono AV, Getzenberg RH, Goebell P, Schmitz-Dräger BJ, et al: Bladder tumour markers beyond cytology: International consensus panel on bladder tumour markers. Urology 66 (6 Suppl 1): 35-63, 2005.

11. D'Costa JJ, Goldsmith JC, Wilson JS, Bryan RT and Ward DG A systematic review of the diagnostic and prognostic value of urinary protein biomarkers in urothelial bladder cancer. Bladder Cancer 2: 301-317, 2016.

12. Garg H, Suri P, Gupta JC, Talwar GP and Dubey S: Survivin: A unique target for tumour therapy. Cancer Cell Int 16: 49, 2016.

13. Jacob NK, Cooley JV, Shirai K and Chakravarti A: Survivin splice variants are not essential for mitotic progression or inhibition of apoptosis induced by doxorubicin and radiation. Onco Targets Ther 5: 7-20, 2012

14. Ambrosini G, Adida C and Altieri DC: A novel anti-apoptosis gene, survivin, expressed in cancer and lymphoma. Nat Med 3 : 917-921, 1997.

15. Swana HS, Grossman D, Anthony JN, Weiss RM and Altieri DC Tumour content of the antiapoptosis molecule survivin and recurrence of bladder cancer. N Engl J Med 341: 452-453, 1999.

16. Smith SD, Wheeler MA, Plescia J, Colberg JW, Weiss RM and Altieri DC: Urine detection of survivin and diagnosis of bladder cancer. JAMA 285: 324-328, 2001

17. Shariat SF, Casella R, Khoddami SM, Hernandez G, Sulser T, Gasser TC and Lerner SP: Urine detection of survivin is a sensitive marker for the noninvasive diagnosis of bladder cancer. J Urol 171: 626-630, 2004.

18. Liang Z, Xin R, Yu Y, Wang R, Wang C and Liu X: Diagnostic value of urinary survivin as a biomarker for bladder cancer: A systematic review and meta-analysis of published studies. World J Urol 36: 1373-1381, 2018

19. Weikert S, Christoph F, Schrader M, Krause H, Miller K and Muller M: Quantitative analysis of survivin mRNA expression in urine and tumour tissue of bladder cancer patients and its potential relevance for disease detection and prognosis. Int J Cancer 116: 100-104, 2005

20. Pu XY, Wang ZP, Chen YR, Wang XH, Wu YL and Wang HP The value of combined use of survivin, cytokeratin 20 and mucin 7 mRNA for bladder cancer detection in voided urine. J Cancer Res Clin Oncol 134: 659-665, 2008.

21. Eissa S, Badr S, Elhamid SA, Helmy AS, Nour M and Esmat M: The value of combined use of survivin mRNA and matrix metalloproteinase 2 and 9 for bladder cancer detection in voided urine Dis Markers 34: 57-62, 2013

22. Moher D, Liberati A, Tetzlaff J, Altman DG and Group P Preferred reporting items for systematic reviews and meta-analyses: The PRISMA statement. PLoS Med 6: e1000097, 2009.

23. Whiting PF, Rutjes AW, Westwood ME, Mallett S, Deeks JJ, Reitsma JB, Leeflang MM, Sterne JA and Bossuyt PM; QUADAS-2 Group: QUADAS-2: A revised tool for the quality assessment of diagnostic accuracy studies. Ann Intern Med 155: $529-536,2011$
24. Skupski DW, Rosenberg CR and Eglinton GS: Intrapartum fetal stimulation tests: A meta-analysis. Obstet Gynecol 99: 129-134, 2002.

25. Deeks JJ, Macaskill P and Irwig L: The performance of tests of publication bias and other sample size effects in systematic reviews of diagnostic test accuracy was assessed. J Clin Epidemiol 58: 882-893, 2005

26. Glas AS, Lijmer JG, Prins MH, Bonsel GJ and Bossuyt PM: The diagnostic odds ratio: A single indicator of test performance. J Clin Epidemiol 56: 1129-1135, 2003.

27. Reitsma JB, Glas AS, Rutjes AW, Scholten RJ, Bossuyt PM and Zwinderman AH: Bivariate analysis of sensitivity and specificity produces informative summary measures in diagnostic reviews. J Clin Epidemiol 58: 982-990, 2005.

28. Higgins JP, Thompson SG, Deeks JJ and Altman DG: Measuring inconsistency in meta-analyses. BMJ 327: 557-560, 2003.

29. Ye X, Xiao H, Chen B and Zhang S: Accuracy of lung ultrasonography versus chest radiography for the diagnosis of adult community-acquired pneumonia: Review of the literature and meta-analysis. PLoS One 10: e0130066, 2015.

30. Al-Maghrebi M, Kehinde EO, Kapila K and Anim JT: Urinary survivin mRNA expression and urinary nuclear matrix protein 22 BladderChek ${ }^{\circledR}$ and urine cytology in the detection of transitional cell carcinoma of the bladder. Med Princ Pract 21: 295-297, 2012.

31. Pina-Cabral L, Santos L, Mesquita B, Amaro T, Magalhães S and Criado B: Detection of survivin mRNA in urine of patients with superficial urothelial cell carcinomas. Clin Transl Oncol 9: 731-736, 2007.

32. Eissa S, Swellam M, Shehata H, El-Khouly IM, El-Zayat T and El-Ahmady O: Expression of HYAL1 and survivin RNA as diagnostic molecular markers for bladder cancer. J Urol 183: 493-498, 2010.

33. Eissa S, Shabayek MI, Ismail MF, El-Allawy RM and Hamdy MA: Diagnostic evaluation of apoptosis inhibitory gene and tissue inhibitor matrix metalloproteinase- 2 in patients with bladder cancer. IUBMB Life 62: 394-399, 2010.

34. Jiang G, Zhang JH, Jian RJ and Chen ZD: Clinical significance of survivin mRNA level and hyaluronic acid level detection of patients suffered from bladder transitional cell carcinomas. Sichuan Med J 11: 1162-1164, 2006.

35. Lin Y, Han ZH and Liu T: The clinical significance of urinary survivin mRNA detection for bladder transitional cell carcinoma. Zhejiang Journal of Integrated Traditional Chinese and Western Medicine 17: 21-22, 2007.

36. Liu JG, Yang JY and Wei W: The value of combined detection of urinary cell keratin 19, nuclear matrix protein 22 and survivin in the early diagnosis of bladder cancer. China Foreign Medical Treatment 28: 168, 2009.

37. Pu XY, Wang ZP, Chen YR, Wu YL, Wang HP and Wang XH: Combined use of uirnary bladder cancer antigen, hyaluronic aeid and survivin for the detection of bladder cancer. Chin J Urol 27: 970-973, 2008.

38. Wan JH, Jin FS, Xiang D, Hu B and Gao F: Value of cytokeratin 20 and survivin in the diagnosis of bladder tumour. J Clin Res 25: 428-431, 2008.

39. Wang L, Zeng FQ, Liao GY and Chen FM: Detection of survivin in exfoliated urothelial cells of bladder cancer. J Clin Urol 19: 489-490, 2004

40. Wang ZH, Hu ZQ, Ye Q, Ye ZQ, Cai D, Yang N, Liu H, Zhuang QY, Yang WM, et al: Clinical application of survivin detection in urothelial cells of patients with transitional cell carcinoma of bladder. Chin J Exper Surg 23: 959-961, 2006.

41. Zhang WX, Zhen S and Zhen T: Diagnosis of bladder cancer by detection of survivin and minichromosome maitence 5 protein in urine sediment. Chin J Urol 26: 233-236, 2005

42. Nicolazzo C, Busetto GM, Del Giudice F, Sperduti I, Giannarelli D, Gradilone A, Gazzaniga P, de Berardinis E and Raimondi $\mathrm{C}$ : The long-term prognostic value of survivin expressing circulating tumor cells in patients with high-risk non-muscle invasive bladder cancer (NMIBC). J Cancer Res Clin Oncol 143: 1971-1976, 2017.

43. Gogalic S, Sauer U, Doppler S and Preininger C: Bladder cancer biomarker array to detect aberrant levels of proteins in urine. Analyst 140: 724-735, 2015.

44. Herr HW: The risk of urinary tract infection after flexible cystoscopy in patients with bladder tumor who did not receive prophylactic antibiotics. J Urol 193: 548-551, 2015.

45. Eissa S, Kassim SK, Labib RA, El-Khouly IM, Ghaffer TM, Sadek M, Razek OA and El-Ahmady O: Detection of bladder carcinoma by combined testing of urine for hyaluronidase and cytokeratin 20 RNAs. Cancer 103: 1356-1362, 2005. 
46. Chang Y, Xu J and Zhang Q: Microplate magnetic chemiluminescence immunoassay for detecting urinary survivin in bladder cancer. Oncol Lett 14: 4043-4052, 2017.

47. Altieri DC: Survivin, versatile modulation of cell division and apoptosis in cancer. Oncogene 22: 8581-8589, 2003

48. Kitsukawa S, Aoyagi T, Noda K, Ito T, Yamamoto Y, Hosoda S, Otsuru N and Matsumoto T: Quantitative analysis of survivin mRNA expression in bladder transitional cell carcinomas. Hinyokika Kiyo 54: 101-106, 2008.
49. Moussa O, Abol-Enein H, Bissada NK, Keane T, Ghoneim MA and Watson DK: Evaluation of survivin reverse transcriptase-polymerase chain reaction for noninvasive detection of bladder cancer. J Urol 175: 2312-2316, 2006.

50. Pormohammad A, Riahi SM, Nasiri MJ, Fallah F, Aghazadeh M, Doustdar F and Pouriran R: Diagnostic test accuracy of adenosine deaminase for tuberculous meningitis: A systematic review and meta-analysis. J Infect 74: 545-554, 2017.

(i) $(9)$ This work is licensed under a Creative Commons Attribution-NonCommercial-NoDerivatives 4.0 International (CC BY-NC-ND 4.0) License. 